\title{
Transgenerational Memory and piRNA
}

\author{
Xinyun $\mathrm{Le}^{1 *}$ \\ ${ }^{1}$ United World College-Changshu China, Suzhou, Jiangsu, 215556, China \\ *Corresponding author. Email: 2213067056@qq.com
}

\begin{abstract}
In this work, we got information from others' papers that memory can be inherited by the next generation, and piRNA is associated with memory inheritance (according to Moore's experiment). PiRNA is found present both in the nervous system and germ cells (according to Kandel's paper) and associated with memory formation (according to Kandel's article). Based on our research, we ask whether it is piRNA that causes memory inheritance. Then, the question comes, can the piRNA associated with the fear-conditioning neuronal circuit in a mouse's brain migrate into their sperm cells? This work hypothesized that if the specific piRNA associated with the fear-conditioning neuronal circuit changes in abundance in the olfactory bulb, the sperm cells would be able to get these piRNA migrated from the brain region and thus inherit the memory of the odour used in the odour fear conditioning. The findings of the research may be applied to diseases prevention.
\end{abstract}

Keywords: Transgenerational memory, piRNA, fear-conditioning

\section{INTRODUCTION}

It's been suggested through several experiments that memory, as well as body traits and diseases could also be inherited via DNA, which is commonly known to carry genetic information.

In Moore's experiment, the result is that "worms can transmit avoidance behavior to their young for four generations, providing progeny with a mechanism to survive dangerous conditions." [1] To see whether memory can be inherited by the next generation, Moore first exposed the worm to a pathogen called Pseudomonas which will attract the worm initially and can make the worm feels sick. After the exposure, the worm started to avoid the pathogen, and the observation shows that the next generation will also prevent the pathogen, the same as the third and the fourth generation. However, until the fifth generation, the worm will no longer prevent the pathogen, and be attracted to the pathogen again, as shown in figure 1. Then, Moore detected whether microRNAs, "[which] are a class of non-coding RNAs that play important roles in regulating gene expression", are related to gene expression, and the result turns out that among the miRNAs, piRNAs change significantly [2]. What's more, the inheritance of memory suggests that piRNAs are present in germ cells.
However, we are not sure whether piRNAs are present in the nervous system. A summary made from Kandel's paper that piRNA can regulate CREB2, as shown from figure 3, which is a transcriptional repressor [3]. PIWI/piRNA complex will bind to the $\mathrm{CpG}$ island on CREB2, using DNMT to methylate the gene. Then, CREB2 is regulated. From this experiment, we get that piRNA is related to memory formation, and present in the nervous system and germ cells. Connecting the result, we get from Kandel's paper and Moore's paper, we made a primary hypothesis that if piRNA can somehow migrate from the nervous system to the germ cells, then the progeny can inherit memories from predecessors.

Inspired by Dias and Ressler's paper, we conducted an odorous fear conditioning (FC) experiment on mice. To know more about the mouse's olfactory system, Sakano's paper shows more clearly how the mouse's olfactory system works, as shown in figure 2. The odorants will first get to the olfactory epithelium (OE). Then the olfactory sensory neuron, which is also the olfactory receptor neuron, will detect the odorant and transmit the odorant to the glomeruli in the olfactory bulb (OB) through the axons of the sensory neuron, with each glomerulus corresponds to one kind of receptor [4].

From these papers, we have already known that memory can be inherited, and is related to piRNAs which are present in the nervous system and germ cells. However, the question is that is the inheritance associated 
with piRNA? We are not sure whether it is piRNA that influences the inheritance of memory because we don't know if the piRNAs exist in germ cells are piRNAs that migrated from the nervous system. Thus, the question will be, can the piRNA associated with the fearconditioning (FC) neuronal circuit in a mouse's brain migrate into their sperm cells? If the specific piRNA associated with the fear-conditioning neuronal circuit changes in abundance in the olfactory bulb, the sperm cells would get these piRNA migrated from the brain region and thus inherit the memory of the odour used in the odour fear conditioning.

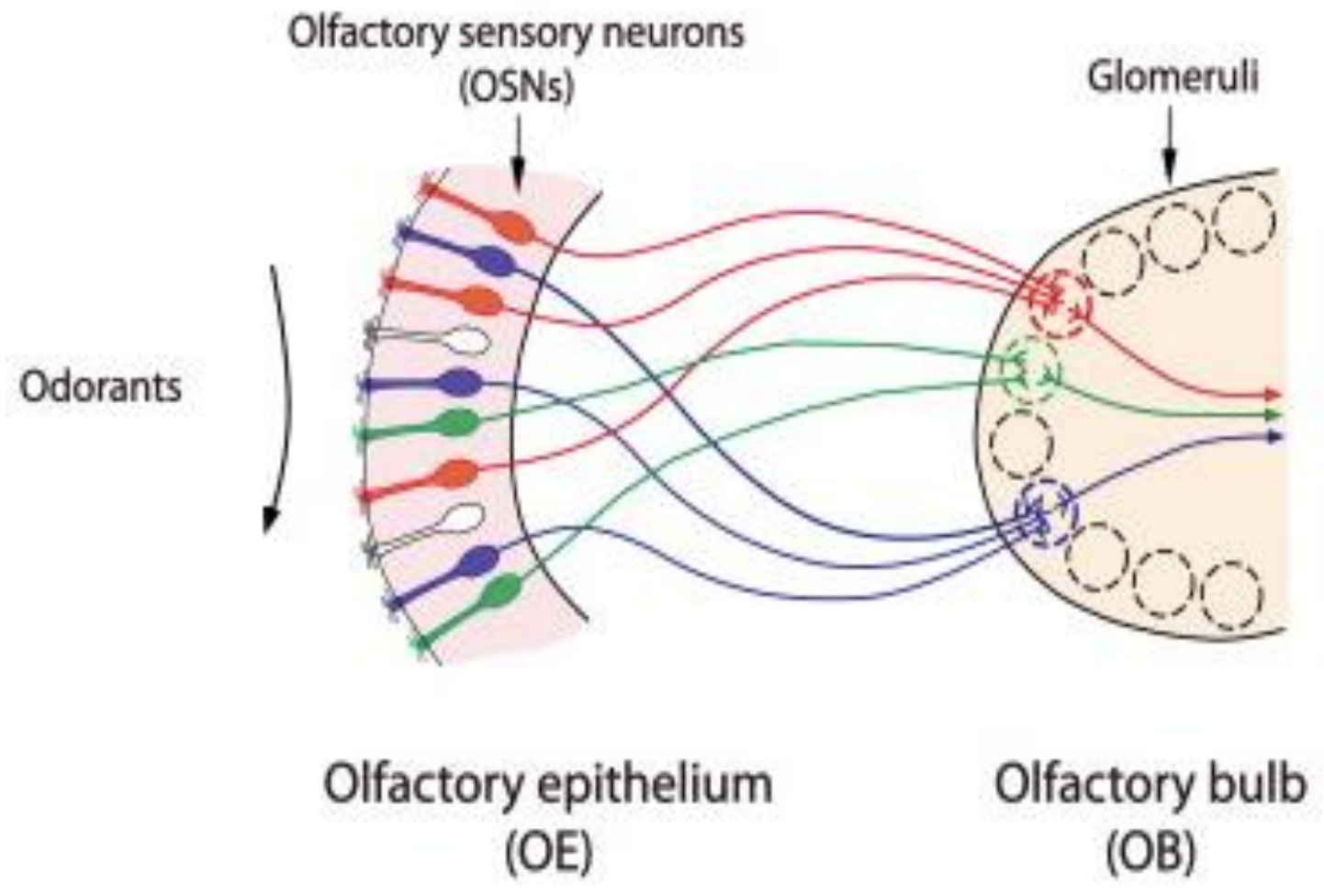

Figure 1 Moore's experiment [1]

\section{Piwi/piRNA complex binds the CREB2 nascent transcript:}

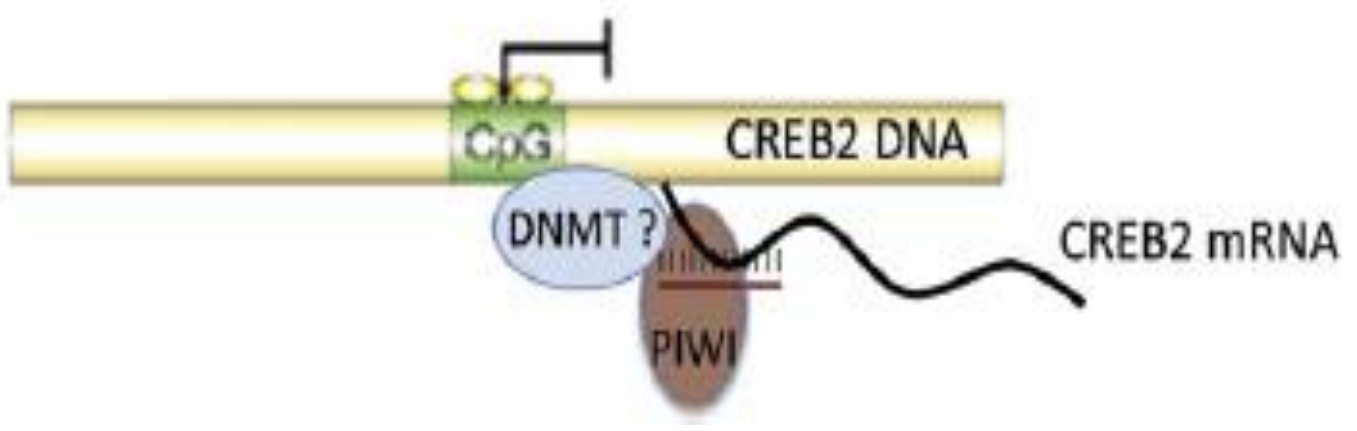

Figure 2 Pathway of odor in the olfactory system [5] 


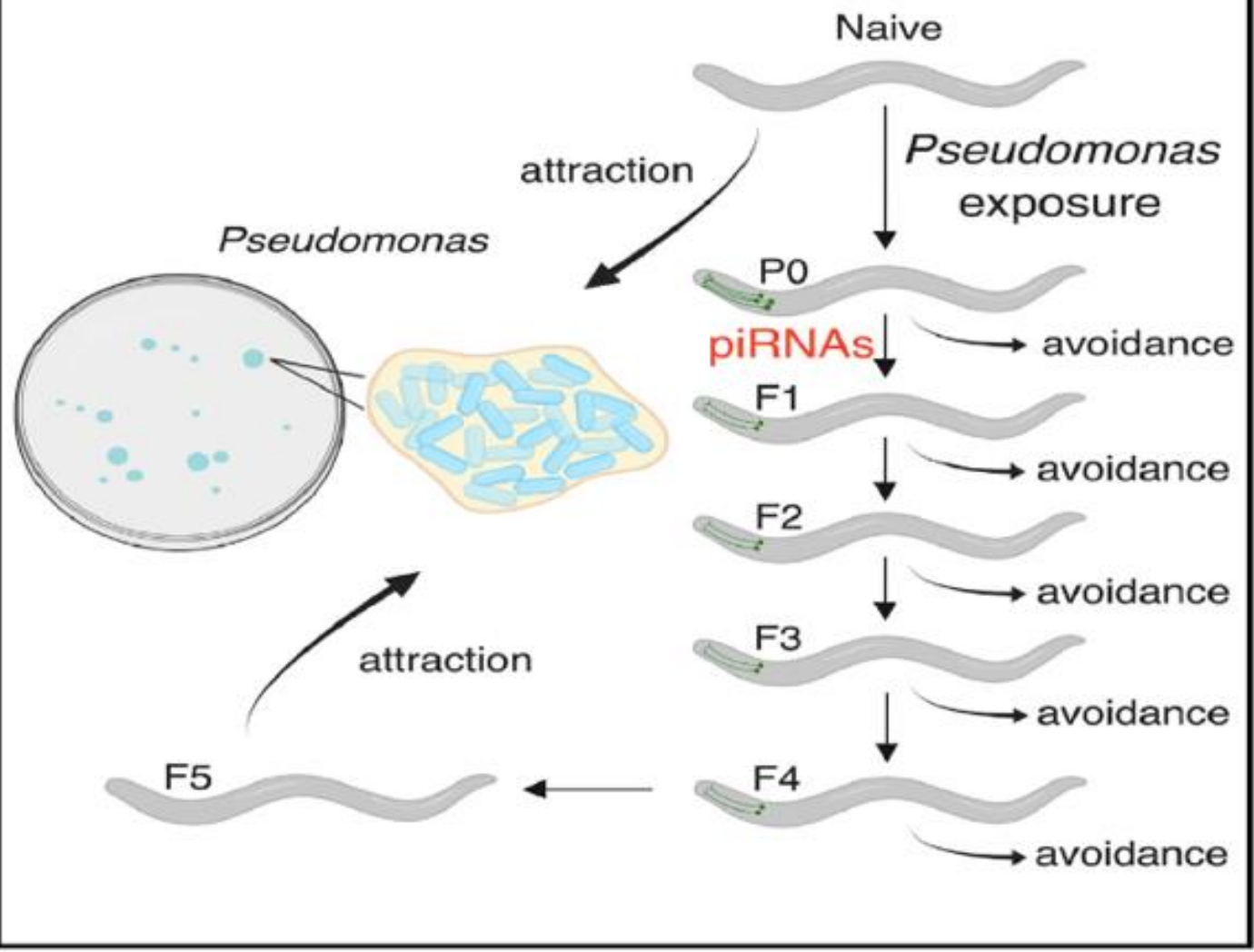

Figure 3 Piwi/piRNA complex binds the CREB2 nascent transcript [6]

\section{EXPERIMENTAL APPROACH}

\subsection{Odor Fear Conditioning on mice}

The mice this work used are adult transgenic mice that contains fluorescent gene on the sensory neurons (M71-the odor receptor of acetophenone) relating to acetophenone sensing so that we can observe the changes of the sensory neurons when conducting FC on mice. Before conducting FC on mice, the mice are put in a circle of 12 hours, light and dark, with free access to food and water. Then we pair the electric shock with odour and check whether mice can discriminate the odour. One experimental group and two control groups will be set up, each consisting of six mice. The experimental group will be treated with electric shock and acetophenone at the same time, control group A will be treated only with acetophenone, and control group B will be treated with electric shock together with propanol. The mice will receive two training sessions per week, lasting three weeks, so that strong odor electric shock association is ensured. Each session consists of five trials of odour conditioning stimulus. After the experiment, we need to check whether the mice would freeze or hide in the corners only when they detect acetophenone. If their freezing behaviour appears when acetophenone is given, the experiment is considered successful, and if not, we need to repeat the experiment.

\subsection{RNA Sequencing and Identification}

To find out the piRNA that is associated with the odor memory, we first need to take out the glomerulus to obtain small RNA clusters. The reason that we only need to take out the glomerulus is that each glomerulus corresponds to one kind of odour. Then we isolate the glomeruli with the neuron's axons and take out the small RNA clusters associated with neuronal circuits. After that, a machine made by Illumina is used to sequence the small RNAs. By using the device, we will be able to detect which RNA is changing most significantly. Later, check if the RNA is piRNA by unique features of piRNA, for example, piRNA has 26-30 nucleotides. The predicted result of the experiment is that there is a significant increase in one of the piRNAs, and we will name it piRNA-X so that we can discriminate it with other piRNAs.

\subsection{Determination}

To determine if the piRNA we've found in RNA sequencing is the correct piRNA, we first will conditionally remove piRNA-X in the OB to see if the piRNA found is indeed required for memory formation. 
Then we knock out the gene that encodes for the piwi protein that works together with the piRNA by CRE recombinase. Later, we repeat $\mathrm{FC}$ conducted on mice. This experiment expects that if the mice will react to the odour after the inhibition of piRNA-X, then piRNA-X is the correct piRNA; if not, then piRNA-X isn't the correct piRNA, and we need to redo the experiment of RNA sequencing.

\subsection{Creation of mutant piRNA}

Since there is a large number of piRNAs in the nervous system, we need to "tag" piRNA-X to see if it appears in the germ cells. Thus, we will use a virus vector to add in a piRNA cluster DNA that has piRNA-X with one mutant nucleotide with a CRE loxP system. Then, introduce the virus to a group of mice embryos. After that, we will use the CRE enzyme in the olfactory bulb region in the adult mice to activate the mutant piRNA, which is the piRNA-X, so that it is expressed in the mice olfactory bulb. A check of mutant piRNA is needed because we need to make sure that the piRNA-X is working. First, we use a cross linker to link piwi protein with mutant piRNA-X to test if they interact. Next, use a specific antibody to separate the piwi-piRNA complex from the cells. Then, elute to separate the piRNA-X and check the presence of mutant piRNA-X with RT-PCR.

\subsection{RT-PCR Test}

RT-PCR stands for reverse transcriptional polymerase chain reaction (RT-PCR) test. It will use the RNA and then reverse transcript it to DNA. To check if piRNA-X is migrated to the sperm cells, we use the RTPCR test to reverse transcript piRNA-X to DNA. The positive result of the RT-PCR test is that the copy of the DNA (cDNA) of the mutant piRNA is found in the sample, then piRNA has migrated from $\mathrm{OB}$ to the germline. A negative result is that cDNA of the mutant piRNA is not found in the sample, then piRNA did not migrate from the olfactory bulb.

\subsection{Odor Fear Conditioning on offspring}

All of the experiments are done to see if piRNA is the factor that causes memory to be inherited. After the investigation, this work checks and see if the offsprings can still inherit the memory. Repeat the same FC experiment conducted on parent generation to the offsprings of the mice of the three groups.

\section{DISCUSSION}

In our experiments, there are still some possible limitations. In RNA sequencing, there could be multiple piRNAs shown to have increased from the baseline mice. In addition, we are not sure whether changing the nucleotides will affect the function of piRNA and consequently damage the mice's ability to form memory. What's more, it is unknown that whether the mutant piRNA will be harmful to the mice.

\section{CONCLUSION}

All the experiments are designed to solve the question that can the piRNA associated with the fear-conditioning neuronal circuit in a mouse's brain migrate into their sperm cells and to test if our hypothesis, which is if the specific piRNA associated with the fear-conditioning neuronal circuit changes in abundance in the $\mathrm{OB}$, the sperm cells would be able to get these piRNA migrated from the brain region and thus inherit the memory of the odour used in the odour fear conditioning, is correct Overall, we have three groups of mice, one is a group of mice with FC and modified piRNA, one is with FC and non-modified piRNA, the other one is without FC and modified piRNA. We first conduct odour fearconditioning on mice, and then we use RNA sequencing to find the piRNA that changes significantly. After that we determine if it is the correct piRNA associated with FC. Then, we create a mutant piRNA for us to detect if it has migrated to the germline, using the RT-PCR test. Finally, we do odour FC on offsprings again to see if it is piRNA that causes memory to inherit.

In conclusion, if mutant piRNA is present in sperm, the piRNA associated with FC has migrated. Thus our hypothesis is accepted. If mutant piRNA is not present in the sperm, then the piRNA associated with FC has not migrated. Thus, our hypothesis is rejected.

However, there are still some questions extended from our study. 1)What is the pathway of migration? What carries the piRNA from the brain to sperms? 2)How did the piRNA pass on to the offsprings and influence their memory? 3)Future studies can also focus on other brain regions related to $\mathrm{FC}$, like the hippocampus and the amygdala.

\section{REFERENCES}

[1] Moore, Rebecca S. et al. "Piwi/PRG-1 Argonaute And TGF-B Mediate Transgenerational Learned Pathogenic Avoidance". Cell, vol 177, no. 7, 2019, pp. 1827-1841.e12. Elsevier BV, doi:10.1016/j.cell.2019.05.024.

[2] O'Brien, Jacob et al. "Overview of Microrna3Biogenesis, Mechanisms Of Actions, And Circulation". Frontiers In Endocrinology, vol 9, 2018. Frontiers Media SA, doi:10.3389/fendo.2018.00402.

[3] Gachon, Frederic et al. "CREB-2, A Cellular CREDependent Transcription Repressor, Functions In Association With Tax As An Activator Of The Human T-Cell Leukemia Virus Type 1 Promoter". Journal of Virology, vol 72, no. 10, 
1998, pp. 8332-8337. American Society For Microbiology, doi:10.1128/jvi.72.10.83328337.1998.

[4] Sakano, Hitoshi. "Neural Map Formation In The Mouse Olfactory System". Neuroscience Research, vol 68, 2010, pp. e11-e12. Elsevier BV, doi:10.1016/j.neures.2010.07.283.

[5] Dias, Brian G, and Kerry J Ressler. "Parental Olfactory Experience Influences Behavior And Neural Structure In Subsequent Generations". Nature Neuroscience, vol 17, no. 1, 2013, pp. 89-96. Springer Science And Business Media LLC, doi:10.1038/nn.3594.

[6] Rajasethupathy, Priyamvada et al. "A Role For Neuronal Pirnas In The Epigenetic Control Of Memory-Related Synaptic Plasticity". Cell, vol 149, no. 3, 2012, pp. 693-707. Elsevier $B V$, doi:10.1016/j.cell.2012.02.057. 